\title{
Isolation and Characterization of Microsatellite Loci in the Chinese Cobra Naja atra (Elapidae)
}

\section{Long-Hui Lin ${ }^{1}$, Lu-Xi Mao ${ }^{2}$, Xia Luo ${ }^{3}$, Yan-Fu Qu ${ }^{2}$ and Xiang Ji ${ }^{2}$ *}

1 Hangzhou Key Laboratory for Animal Adaptation and Evolution, School of Life Sciences, Hangzhou Normal University, Hangzhou 310036, Zhejiang, China; E-Mail: linlh@yahoo.cn

2 Jiangsu Key Laboratory for Biodiversity and Biotechnology, College of Life Sciences, Nanjing Normal University, Nanjing 210046, Jiangsu, China; E-Mails: maoluxi@ hotmail.com (L.-X.M.); imwx-q@163.com (Y.-F.Q.)

3 School of Life Science, Anhui University, Hefei 230039, Anhui, China;

E-Mail: 1xsm1986@163.com

* Author to whom correspondence should be addressed; E-Mail: xji@ mail.hz.zj.cn; Tel.: +86-25-85891597; Fax: +86-25-85891526.

Received: 7 June 2011; in revised form: 1 July 2011 / Accepted: 4 July 2011 / Published: 7 July 2011

\begin{abstract}
We characterize thirteen polymorphic microsatellite loci isolated from Naja atra genomic libraries, which were enriched for AC-motif microsatellites. The thirteen loci were screened on a group of 48 individuals from two populations, one in Yong'an and the other in Ganzhou. These markers revealed a relatively high degree of genetic diversity (4-12 alleles per locus) and heterozygosity (Ho ranged from $0.213-0.854$ and He ranged from 0.301-0.838). Tests for departure from Hardy-Weinberg equilibrium and for linkage disequilibrium were conducted for each of the two populations separately. After sequential Bonferroni correction, none of the 13 loci showed significant departures from Hardy-Weinberg equilibrium. Hierarchical analysis of molecular variance indicated that a small but significant $(P<0.001)$ proportion $(16.0 \%)$ of the total variation in the microsatellite DNA data were attributable to differences among populations, indicating geographical structuring and restricted gene flow. It could be attributable to the Wuyi mountains in the area having a sufficiently isolating effect to significantly reduce gene flow. Our microsatellite data also showed a low $N_{\mathrm{m}}$ (1.31) value in the two populations from mainland China. Thus, the Yong'an and Ganzhou populations could be treated as distinct evolutionarily significant units (ESUs). The high level of polymorphism revealed
\end{abstract}


by these microsatellite markers will be useful for the study of gene flow, population structure and evolutionary history of $N$. atra.

Keywords: Naja atra; Elapidae; microsatellites; PCR primers; polymorphism

\section{Introduction}

The Chinese cobra (Naja atra) is an oviparous elapid snake with a distribution covering southeastern China (including Taiwan, Hongkong and Hainan) and northern Vietnam (see Figure 1) [1]. Naja atra was one of the snakes most commonly found in China thirty years ago. Nowadays, it is regarded as a highly vulnerable species according to the China Red Data Book of Endangered Animals [2], largely because cobras have been over-hunted by local people for their meat and skin, which are used in medicine and handwork. There are realistic threats of local extinction in some southern provinces such as Guangdong and Hainan [2]. To develop effective conservation strategies for the cobra, we need a better understanding of gene flow, population structure and evolutionary history of this species. One approach for achieving this goal is to use specific molecular markers. Among them, microsatellites or short, tandemly repeated base-pair sequences are the most useful due to the high variability caused by changes in their repeat number [3]. Here, we describe the isolation and characterization of thirteen microsatellite markers in N. atra.

Figure 1. Map of Southeast China, showing the two localities where the cobras used in this study were collected. GZ: Ganzhou; YA: Yong'an.

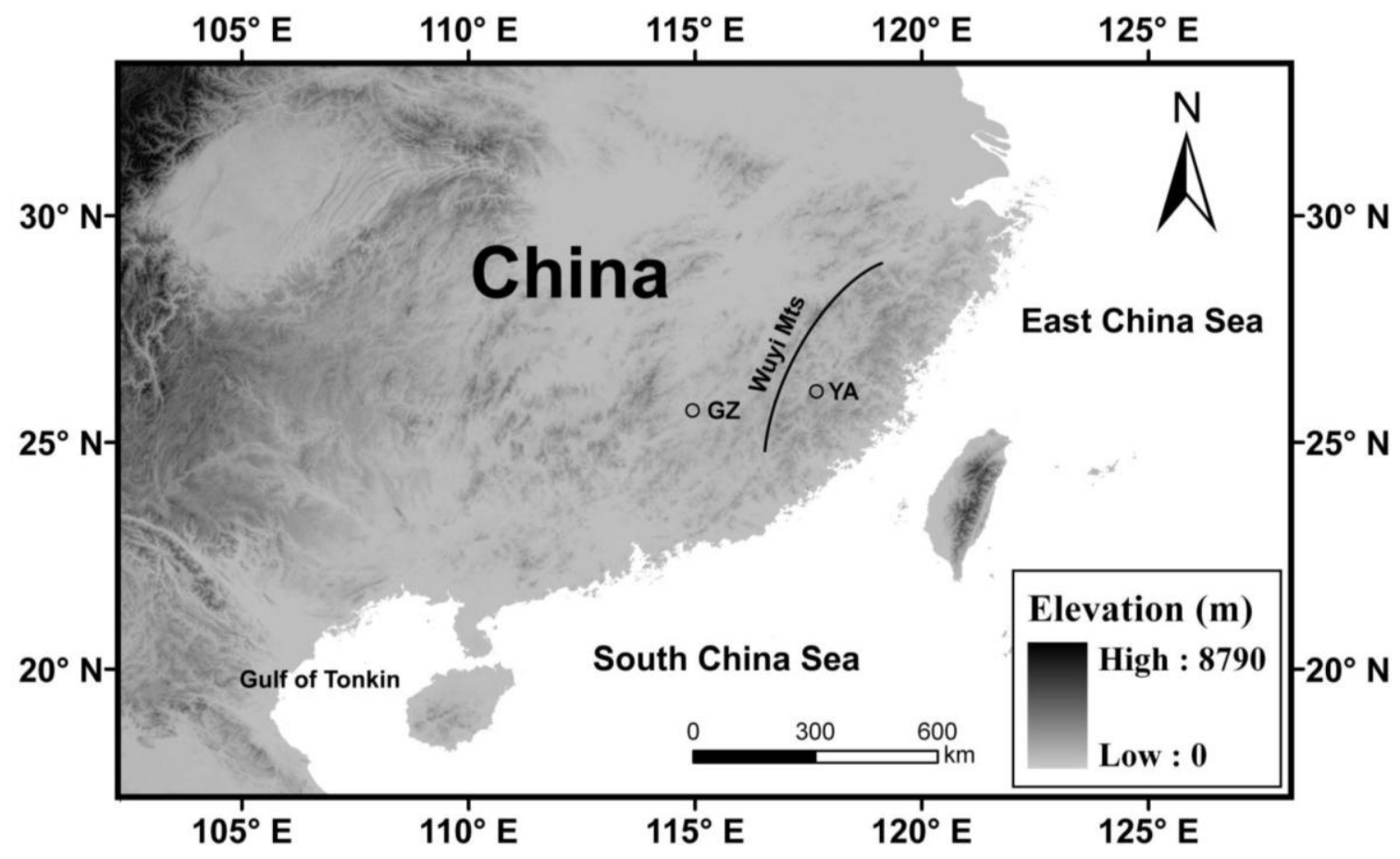




\section{Material and Methods}

Forty-eight individuals ( $n=24$ for each population) used in this study were collected from two populations, one in Yong'an (Fujian Province) and the other in Ganzhou (Jiangxi Province). Fresh tissue (tail muscle) samples were used to extract total genomic DNA using EasyPure Genomic DNA Extraction Kit (TransGen Biotech). DNA was resuspended in TE buffer (10 mM Tris-HCl, pH 8.0, $0.1 \mathrm{mM}$ EDTA). Genomic DNA was used to construct a library enriched for AC-motif microsatellite sequences. The enrichment protocol essentially followed the FIASCO (Fast Isolation by AFLP of sequences COtaning repeats) [4] with minor modifications. Genomic DNA was digested with Mse I and ligated to Mse I AFLP adaptor. The digestion-ligation mixture was diluted (1:10), and directly amplified in a total volume of $20 \mu \mathrm{L}$ with primer Mse I-N. The product from this step was mixed $1 \mu \mathrm{L}$ (about $20 \mathrm{pmol}$ ) of a 5'-biotinylated (AC) 10 oligonucleotide probe in a total volume of $40 \mu \mathrm{L}$ $(0.1 \times$ saline sodium citrate buffer $)$ at $68^{\circ} \mathrm{C}$ for $1 \mathrm{~h}$. The DNA fragment $(\mathrm{AC})_{10}$ complex were obtained by adsorption of streptavidin and separated by a magnetic field from the hybridization buffer. The complex fragments were ligated into PMD19-T vector and transformed into competent cells (DH5 $\alpha$ ). Cells were plated on "blue-white" of Luria-Bertani plates. In total, 384 positive colonies were selected by polymerase chain reaction using nonbiotinylated $(\mathrm{AC})_{10}$ probe primer and universal M13 primers. Those that produced a two-band pattern in agarose electrophoresis were considered to contain a microsatellite repeat.

For the microsatellites sequences containing adequate flanking regions, PCR primers were designed using CID software [5]. From these primers, 28 produced consistent amplification of loci in eight tested individuals fromYong'an population. All PCRs were performed with the following conditions (in 20- $\mu \mathrm{L}$ volume): 100-200 ng of genomic DNA, $10 \mu \mathrm{L} 2 \times$ EasyTaq PCR SuperMix polymerase (TransGen Biotech), $0.5 \mu \mathrm{M}$ of each primer; 5 min denaturation at $95{ }^{\circ} \mathrm{C} ; 32$ cycles of $30 \mathrm{~s}$ at $95{ }^{\circ} \mathrm{C}$, $1 \mathrm{~min}$ at specific annealing temperatures, and $1 \mathrm{~min}$ at $72{ }^{\circ} \mathrm{C}$ (Table 1); and a final extension of $72{ }^{\circ} \mathrm{C}$ for $10 \mathrm{~min}$. Amplification and polymorphism were assessed in 48 individuals of $N$. atra from the Yong'an and Ganzhou populations. All PCR products were genotyped using an ABI PRISM 3730 genetic analyzer (Applied Biosystems) along with Rox GS350 size standard, and analyzed with GENEMARKER (version 1.85, Applied Biosystems).

The number of alleles at each polymorphic locus, their size range and observed and expected heterozygosities were calculated using CERVUS 3.0 software [6]. Deviation from Hardy-Weinberg equilibrium (HWE) and linkage disequilibrium (LD) at each locus was calculated using GENEPOP 4.0.10 [7,8]. Hierarchical analysis of molecular variance (AMOVA) with 1,000 permutations was performed to examine partitioning of genetic diversity within and among populations using ARLEQUIN 3.11 [9].

\section{Results and Discussion}

Details of the microsatellite loci and variability measures across 48 individuals of the Chinese cobra are summarized in Table 1. The 13 sequences containing a microsatellite locus were deposited in GenBank (HQ881484-HQ881496). The number of alleles ranged from 4 to 12; observed heterozygosity (Ho) ranged from 0.213-0.854, and expected heterozygosity (He) ranged from 0.301-0.838 (Table 1). 
Table 1. Characteristics of 13 Naja atra microsatellites DNA loci: locus designation, repeat motif, primer sequences, allele size range (bp), annealing temperature, $T_{a}\left({ }^{\circ} \mathrm{C}\right)$, observed number of alleles $\left(N_{a}\right)$ and effective number of alleles $\left(N_{e}\right)$, average observed $\left(H_{o}\right)$ and expected heterozygosity $\left(H_{e}\right)$, Polymorphic information content $(P I C)$, number of individuals successfully genotyped $(N)$, and GenBank Accession number.

\begin{tabular}{|c|c|c|c|c|c|c|c|c|c|c|c|}
\hline Locus & Repeat motif & Primer sequences (5'-3') & Size range (bp) & $T_{a}\left({ }^{\circ} \mathbf{C}\right)$ & $N_{a}$ & $N_{e}$ & $\mathrm{H}_{o}$ & $\boldsymbol{H}_{e}$ & PIC & $N$ & Accession No. \\
\hline \multirow[t]{2}{*}{ p26 } & $(\text { TGTA })_{11}$ & F:5' TAMRA-TGCGAAATAACCCATTCTAATC & $200-242$ & 55 & 9 & 4.378 & 0.525 & 0.781 & 0.743 & 40 & HQ881486 \\
\hline & & R:5’-TCTAGATGCTTATATGACCACAGG & & & & & & & & & \\
\hline \multirow[t]{2}{*}{ p92 } & $(\mathrm{AC})_{14}$ & F:5' HEX-AACCTCTCCCTGAATCAACTAG & $145-171$ & 55 & 7 & 2.344 & 0.512 & 0.580 & 0.492 & 43 & HQ881492 \\
\hline & & R:5'-GCAGATATAGCACACTTGTTTG & & & & & & & & & \\
\hline \multirow[t]{2}{*}{ p122 } & $(\mathrm{AC})_{16}$ & F:5' FAM-TGAATGAAGGAACAGCACAATC & $140-170$ & 55 & 4 & 2.090 & 0.500 & 0.527 & 0.461 & 44 & HQ881490 \\
\hline & & R:5'-AACAGGAGCGAAGAGGTTTC & & & & & & & & & \\
\hline \multirow[t]{2}{*}{$\mathrm{p} 121$} & $(\mathrm{GT})_{18}$ & F:5' TAMRA-AGTCAACTGAATCTTCTCCATC & 173-208 & 55 & 6 & 2.620 & 0.213 & 0.625 & 0.590 & 47 & HQ881493 \\
\hline & & R: 5'-CAACTCCACTTCCCCAGAC & & & & & & & & & \\
\hline \multirow[t]{2}{*}{$\mathrm{p} 8$} & $(\mathrm{GT})_{15}$ & F:5' FAM-TTCATTGAGTGGTGAGTGAGC & 118-146 & 55 & 8 & 2.012 & 0.362 & 0.508 & 0.460 & 47 & HQ881488 \\
\hline & & R:5'-CTTCAGACCCTTCTCTTTATTACTC & & & & & & & & & \\
\hline \multirow[t]{2}{*}{ p124 } & $(\mathrm{CA})_{19}$ & F:5' TAMRA-CACTGTTGGTCTGAAAATCTCTAT & $103-140$ & 55 & 10 & 5.863 & 0.417 & 0.838 & 0.807 & 48 & HQ881487 \\
\hline & & R:5'-GTTTTCAGTTTGTTTGTCAATGTC & & & & & & & & & \\
\hline \multirow[t]{2}{*}{$\mathrm{p} 88$} & $(\mathrm{AC})_{14}$ & F:5' HEX-CACACAAACAATCGCTCTACAC & $50-77$ & 55 & 6 & 1.936 & 0.396 & 0.489 & 0.454 & 48 & HQ881484 \\
\hline & & R:5’-AGCCTTCCAGACATCAAATCAG & & & & & & & & & \\
\hline \multirow[t]{2}{*}{ p87 } & $(\mathrm{GT})_{22}$ & F: 5' FAM-AAATCCCAGTAGAGGTTTGTTC & $102-144$ & 55 & 8 & 4.267 & 0.417 & 0.774 & 0.732 & 48 & HQ881489 \\
\hline & & R: 5'-GGACAGTGGTCGTCACAC & & & & & & & & & \\
\hline \multirow[t]{2}{*}{ p265 } & $(\mathrm{TG})_{19}$ & F:5' HEX-GCACACAGGGTCTTCATTATTG & $128-164$ & 55 & 10 & 5.260 & 0.646 & 0.818 & 0.785 & 48 & HQ881491 \\
\hline & & R:5'-TCCACAGCGAAACTCATCAG & & & & & & & & & \\
\hline \multirow[t]{2}{*}{ p132 } & $(\mathrm{AC})_{20}$ & F: 5' FAM-TTCACCAGGTTCCAATGTTCC & $83-121$ & 56 & 5 & 1.425 & 0.319 & 0.301 & 0.280 & 47 & HQ881485 \\
\hline & & R: 5'-AGTCAGCCATTGTTCACTCTG & & & & & & & & & \\
\hline \multirow[t]{2}{*}{$\mathrm{p} 22$} & $(\mathrm{CA})_{22}$ & F: 5' TAMRA-CAAGGGGACAGTGGTCTTC & $52-94$ & 55 & 6 & 2.628 & 0.468 & 0.626 & 0.563 & 47 & HQ881494 \\
\hline & & R: 5'- TCTGGGCAAATGATGAAAATCC & & & & & & & & & \\
\hline \multirow[t]{2}{*}{ p262 } & $(\mathrm{AC})_{16}$ & F:5' HEX-CACTGGAGCTGGACACTTG & $107-137$ & 55 & 12 & 5.804 & 0.854 & 0.836 & 0.810 & 48 & HQ881495 \\
\hline & & R:5'-TCATTTAGAGCAAAGGTGATGC & & & & & & & & & \\
\hline \multirow[t]{2}{*}{ p140 } & $(\mathrm{TG})_{16}$ & F:5' FAM-GGAAAGATGGAAATGGCTTCAC & $142-173$ & 55 & 7 & 2.122 & 0.333 & 0.534 & 0.493 & 48 & HQ881496 \\
\hline & & R:5'-ATTCGGTTTGGTGGCAGAC & & & & & & & & & \\
\hline
\end{tabular}


Tests for departure from HWE and for LD were conducted for each of the two populations separately. After sequential Bonferroni correction [10], none of the 13 loci showed significant departures from Hardy-Weinberg equilibrium for each of the two populations. Two (from the Yong'an population) and one (from the Ganzhou population) out of 78 pairwise comparisons exhibited significant linkage disequilibrium respectively following sequential Bonferroni correction.

AMOVA indicated that a small but significant $(P<0.001)$ proportion $(16.0 \%)$ of the total variation in the microsatellite DNA data were attributable to differences among populations, indicating geographical structuring and restricted gene flow. It could be attributable to the Wuyi mountains in the area having a sufficiently isolating effect to significantly reduce gene flow (see Figure 1). A significant $(P<0.001)$ proportion of variation also occurred within populations $(84.0 \%)$. Previous work based on data with the mitochondrial control region sequences of the Chinese cobra in Taiwan showed low $N_{\text {m }}$ values (0.31-2.00) between populations, indicating low levels of gene flow [11]. Accordingly, the four populations in Taiwan were treated as distinct evolutionarily significant units (ESUs) [11]. Our microsatellite data also showed a low $N_{\mathrm{m}}(1.31)$ value between the two populations from mainland China. The results of AMOVA and the low $N_{\mathrm{m}}$ value between the two populations indicated that the Yong'an and Ganzhou populations should be treated as distinct ESUs. Given the existence of distinct ESUs, reintroducing confiscated cobras from the illegal trade back into the wild should be conducted with caution to prevent artificial gene flow.

In conclusion, the microsatellite loci presented in this study will be useful for genetic studies in $N$. atra especially directed toward a finer-scaled understanding of gene flow, population structure and dispersal dynamics within and between population groups.

\section{Acknowledgments}

This work was supported by grants from National Natural Science Foundation of China (Project No. 31070339), Zhejiang Provincial Foundation of Natural Science (Project No. Z3090461) and Hangzhou Bureau of Science and Technology (Project No. 20100332T20). We thank Bao-Wei Zhang, Lian Chen, Hong Li, Qian Chen and Hui Wang for their help during the research.

\section{References}

1. Wüster, W.; Golay, P.; Warrell, D.A. Synopsis of recent developments in venomous snake systematics. Toxicon 1997, 35, 319-340.

2. Zhao, E.M. Naja atra cantor. In China Red Data Book of Endangered Animals (Amphibia and Reptilia); Zhao, E.M., Ed.; Science Press: Beijing, China, 1998; pp. 274-276 (in Chinese).

3. Schlötterer, C.; Pemberton, J. The use of microsatellites for genetic analysis of natural populations-A critical review. In Molecular Approaches to Ecology and Evolution; Desale, R., Schierwater, B., Eds.; Birkhäuser Verlag: Basel, Switzerland, 1998; pp. 71-86.

4. Zane, L.; Bargelleoni, L.; Patarenello, T. Strategies for microsatellite isolation: A review. Mol. Ecol. 2002, 11, 1-16.

5. Freitas, P.D.; Martins, D.S.; Galettijr, P.M. CID: A rapid and efficient bioinformatic tool for the detection of SSRs from genomic libraries. Mol. Ecol. Resour. 2008, 8, 107-108. 
6. Kalinowski, S.T.; Taper, M.L.; Marshall, T.C. Revising how the computer program CERVUS accommodates genotyping error increases success in paternity assignment. Mol. Ecol. 2007, 16, 1099-1106.

7. Raymond, M.; Rousset, F. GENEPOP (version 1.2): Population genetics software for exact tests and ecumenicism. J. Hered. 1995, 86, 248-249.

8. Rousset, F. Genepop'007: A complete reimplementation of the Genepop software for Windows and Linux. Mol. Ecol. Resour. 2008, 8, 103-106.

9. Excoffier, L.; Laval, G.; Schneider, S. ARLEQUIN (version 3.0): An integrated software package for population genetics data analysis. Evol. Bioinf. Online 2005, 1, 47-50.

10. Rice, W.R. Analyzing tables of statistical tests. Evolution 1989, 43, 223-225.

11. Lin, H.C.; Li, S.H.; Fong, J. Ventral coloration differentiation and mitochondrial sequences of the Chinese cobra (Naja atra) in Taiwan. Conserv. Genet. 2008, 9, 1089-1097.

(C) 2011 by the authors; licensee MDPI, Basel, Switzerland. This article is an open access article distributed under the terms and conditions of the Creative Commons Attribution license (http://creativecommons.org/licenses/by/3.0/). 